\title{
Improved 3-omega measurement of thermal conductivity in liquid, gases, and powders using a metal-coated optical fiber
}

\author{
Scott N. Schiffres and Jonathan A. Malen a) \\ Carnegie Mellon University, Pittsburgh, Pennsylvania 15213, USA
}

(Received 9 December 2010; accepted 28 April 2011; published online 10 June 2011)

\begin{abstract}
A novel $3 \omega$ thermal conductivity measurement technique called metal-coated $3 \omega$ is introduced for use with liquids, gases, powders, and aerogels. This technique employs a micron-scale metal-coated glass fiber as a heater/thermometer that is suspended within the sample. Metal-coated $3 \omega$ exceeds alternate $3 \omega$ based fluid sensing techniques in a number of key metrics enabling rapid measurements of small samples of materials with very low thermal effusivity (gases), using smaller temperature oscillations with lower parasitic conduction losses. Its advantages relative to existing fluid measurement techniques, including transient hot-wire, steady-state methods, and solid-wire $3 \omega$ are discussed. A generalized $n$-layer concentric cylindrical periodic heating solution that accounts for thermal boundary resistance is presented. Improved sensitivity to boundary conductance is recognized through this model. Metal-coated $3 \omega$ was successfully validated through a benchmark study of gases and liquids spanning two-orders of magnitude in thermal conductivity. (C) 2011 American Institute of Physics. [doi:10.1063/1.3593372]
\end{abstract}

\section{INTRODUCTION}

While thermal transport properties have been measured for centuries, measurement techniques for thermal conductivity have revived importance due to the emergence of novel and challenging to measure materials, including thin-films, superlattices, biological samples, and nanomaterials. ${ }^{1-10}$ Enhanced thermal property information will aid a diverse range of applications, including improved microprocessor heat removal, ${ }^{11,12}$ higher efficiency thermoelectric materials, ${ }^{13,14}$ and more effective minimally invasive cryosurgery. ${ }^{15}$ The $3 \omega$ method and time domain thermo-reflectance (TDTR) are the dominant tools for measuring thermal conductivity in solids, while fluid samples have typically been studied with transient hot-wire (THW). Recent studies have used modified versions of $3 \omega$ and TDTR to measure fluid samples. ${ }^{9,16}$ Herein we introduce and validate a modified $3 \omega$ method for fluid measurement using a metal-coated glass fiber as a heater/thermometer. This technique, unlike prior $3 \omega$ incarnations, is sensitive to liquids, gases, powders, and aerogels.

Current methods for sensing fluid thermal properties can be categorized by the thermal forcing function: steadystate, impulse, or periodic. Steady-state thermal property techniques apply a known one-dimensional steady heat flux and measure the temperature versus distance. The thermal conductivity is the quotient of heat flux per unit area and the slope of temperature change with distance. Steady-state techniques have serious drawbacks that include long settling times, natural convection effects, radiation losses, complex 3D isotherms, and relatively large test samples. ${ }^{17-19}$ The most widely used steady-state technique, guarded hot plate, often uses meter squared test samples. ${ }^{19}$

\footnotetext{
a) Author to whom correspondence should be addressed. Electronic mail:
} jonmalen@cmu.edu.
While steady-state techniques are conceptually simple, accurate implementation is fraught with difficulties due to these factors.

Amongst non-steady state techniques for fluid property measurement, THW dominates. ${ }^{4,8,20}$ THW analyzes the thermal impulse signal in the time domain, rather than in the frequency domain. THW sends a thermal impulse from a thin heating wire into the surrounding fluid of interest. Simultaneously, the resistance of the wire increases with the wire's increasing temperature, and the coefficient of thermoresistance characterizes the resistance change with temperature. Therefore, the measured resistance corresponds to the wire temperature. From the time-resolved wire temperature, the thermal conductivity of the surrounding fluid can be determined. ${ }^{21,22}$ Because the heating step-impulse contains signals at all frequencies, the data used to determine thermal conductivity will contain long wavelength heat-waves. These long thermal waves decay in amplitude slowly with increasing depth (long thermal penetration depths), and so will be most affected by the container dimensions, especially as time progresses. Because these long-wavelength components of the heat impulse cannot be excluded from the thermal impulse, they have greater sensitivity to the test vessel boundaries than frequency-domain techniques. Hence, THW requires either truncating data sets to consider only very short times with compromised accuracy, or the use of a bigger fluid sample relative to frequency domain measurements. This increased fluid requirement is a disadvantage, particularly when the fluid under test cannot be synthesized in large quantities, such as biological fluid samples.

Frequency domain based $3 \omega$ techniques use a resistance heater/thermometer to periodically heat a sample and simultaneously monitor its thermal response. For solids, the heater/thermometer is a thin metal strip that is microfabricated on the sample being tested (see Fig. 1(a)). The alternating joule heating applied to the metal strip has current 

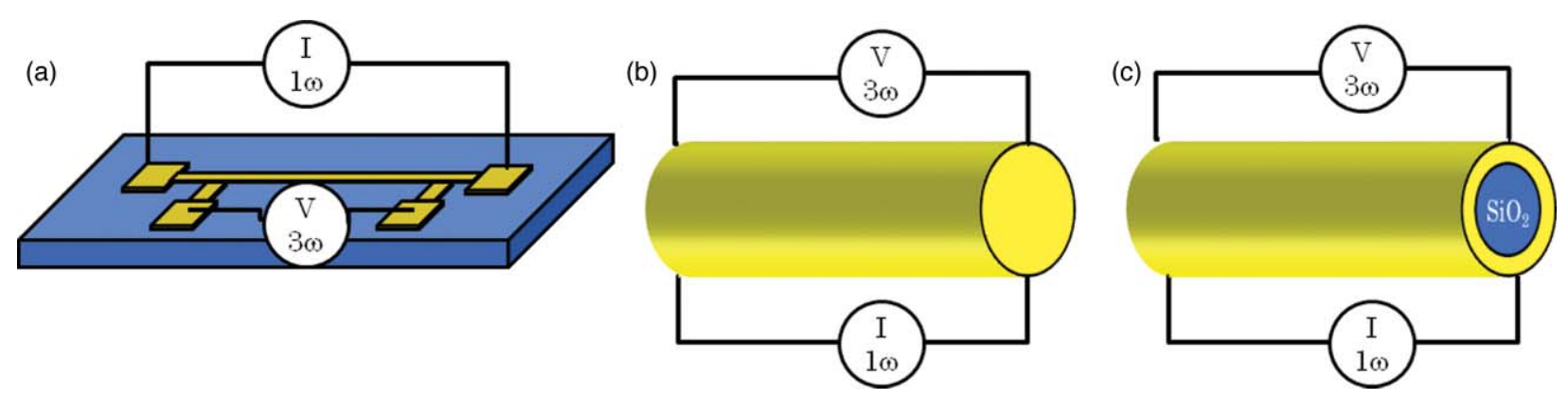

FIG. 1. (Color online) Comparison of three $3 \omega$ techniques: (a) Standard $3 \omega$-heater is built on solid substrate. The fluid to be sensed is above the heater. The outer probes drive a $1 \omega$ current and the inner probes sense a third-harmonic voltage signal $3 \omega$. (b) Solid-wire $3 \omega-$ has the $1 \omega$ current driven through the length of the wire and $3 \omega$ voltage sensed across the length of the wire. The fluid to be measured surrounds the fiber. (c) Metal-coated $3 \omega-$ employs a glass fiber as a backbone for a nanometer thick metal coating. The voltage and current is applied similar to solid metal wire, with the fluid surrounding the metal coating.

periodically passing through it at a frequency of $1 \omega$. Because joule heating is a quadratic function of current $\left(P=I^{2} R\right)$, the heating occurs at twice the current drive frequency, at $2 \omega$. This periodic heating at $2 \omega$ generates periodic traveling thermal waves that decay in amplitude with increasing depth into the surrounding medium. The thermophysical properties of the surrounding medium will affect the amplitude and phase of the temperature oscillations in the heating line in a way that can be predicted using analytical or numerical conduction models. ${ }^{23,24}$

The amplitude will exponentially decay into the solid, with a characteristic length scale referred to as, the thermal penetration depth $\left(L_{P}\right)$. Thermal penetration depth is a function of the thermal diffusivity $\alpha$ of the material, and the current's angular frequency $\omega\left(L_{p}=\sqrt{\alpha / \omega}\right)$. As the drive frequency is swept from low to high-frequency, the thermal penetration depth shrinks. Likewise, the magnitude of the temperature oscillations in the heating line will vary with driving frequency. Large temperature oscillations occur at low-frequency, while small temperature oscillations occur at high-frequency. This temperature versus frequency behavior depends on thermal conductivity and therefore thermal conductivity can be determined from this information. As the temperature in the wire oscillates at $2 \omega$, so does the resistance, as quantified by the coefficient of thermoresistance $\left(\beta=\frac{1}{R_{E l}} \frac{d R_{E l}}{d T}\right)$. The voltage, the product of current, and resistance, will have a component at 3 times the current frequency $(3 \omega)$ stemming from product the $1 \omega$ current and $2 \omega$ resistance signals. $^{23}$

When the standard $3 \omega$ is used to measure fluids, the heater is patterned on a solid and the fluid is placed over this heater. A significant disadvantage of standard $3 \omega$ for measuring fluid properties is that the solid substrate typically has much larger thermal effusivity $\kappa \rho c_{p}$, than a fluid. Because the heat generated in the metal strip will split between the solid substrate and the fluid based on their ratio of thermal effusivities ${ }^{25}$ much of the heat will enter the substrate rather than the fluid. For example, for a standard $3 \omega$ experiment built on a Pyrex substrate measuring air at standard temperature and pressure, greater than 250 units of heat will enter the Pyrex for every unit of heat entering the air. Consequently, a significant part of the $3 \omega$ signal comes from the substrate, rather than the fluid under test, which decreases the signal of interest's size. ${ }^{4}$ Solid-wire $3 \omega$ uses a solid heating wire that is immersed within the sample, rather than a microfabricated heater on a planar substrate. Similar to the standard $3 \omega$, much of the periodic heating propagates radially into the high thermal effusivity metal wire, rather than into surrounding fluid.

\section{A. Description of metal-coated immersed wire 3-omega}

Metal-coated immersed wire $3 \omega$ enhances prior fluid measurement techniques by advancing the beneficial qualities of THW, solid-wire $3 \omega$, and standard $3 \omega \cdot{ }^{3,7,9,26}$ Metal-coated $3 \omega$ improves THW and solid-wire $3 \omega$ 's heater by using a micron-scale metal-coated optical fiber rather than a thin solid metal wire. The thinner metal-cross-section results in signal improvements due to increased axial electrical and thermal resistance. Increased electrical resistance leads to a larger $3 \omega$ signal, which enhances the experimental signal to noise ratio. Increased axial thermal resistance reduces the boundary effects of the vessel, by reducing the thermal conductivity of the heating element. Further, with metal-coated $3 \omega$, less of this periodic heat is parasitically lost into the low thermal effusivity glass fiber, which leads to greater sensitivity to the thermal conductivity of the surrounding fluid. Unlike THW, metal-coated $3 \omega$ operates in the frequency domain, allowing control of $L_{p}$ to accommodate small samples. Finally, simulations with conductance models show an increased sensitivity to thermal boundary resistance. These signal improvements have a tangible effect, thus making possible measurements of low thermal effusivity materials that have been hitherto impossible with $3 \omega$ techniques. ${ }^{10}$

\section{B. Quantitative analysis of the relative advantages of metal-coated $3 \omega$}

The 3rd harmonic voltage signal strength per temperature oscillation intensity, $\Delta V_{3 \omega} / \Delta T_{2 \omega}$, characterizes a key performance benefit of metal-coated $3 \omega$ relative to prior solid-wire $3 \omega$ techniques. Larger $\Delta V_{3 \omega} / \Delta T_{2 \omega}$ enables thermal property measurement with smaller $\Delta T_{2 \omega}$,

$$
\frac{\Delta V_{3 \omega}}{\Delta T_{2 \omega}}=\frac{\Delta R_{2 \omega} I_{1 w}}{\Delta T_{2 \omega}}=\beta R_{E l} I_{1 w} .
$$


To maintain a given $\Delta T_{2 \omega}$ the current and resistance must vary such that power dissipated, $I_{1 \omega}^{2} R_{E l}$, remains constant. Therefore $I_{1 \omega}$ must vary as $\left(\sqrt{R_{E l}}\right)^{-1}$. Additionally, $R_{E l}$ varies inversely with the cross-sectional area of the metal heating layer, $A_{H}$,

$$
\frac{\Delta V_{3 \omega}}{\Delta T_{2 \omega}} \propto \sqrt{R_{E l}} \propto \frac{1}{\sqrt{A_{H}}} .
$$

Relative to solid metal wires, the thin metal film coating can have more than 2 orders of magnitude smaller $A_{H}$. The below expression compares the signal to temperature oscillation magnitude for metal-coated $3 \omega$ to solid metal $3 \omega$, provided they are measuring the same fluid and the heating layer is the same metal,

$$
\frac{\left(\Delta V_{3 \omega} / \Delta T_{2 \omega}\right)_{M C}}{\left(\Delta V_{3 \omega} / \Delta T_{2 \omega}\right)_{S W}} \propto \frac{\sqrt{A_{H, S W}}}{\sqrt{A_{H, M C}}} .
$$

For instance, the approximate signal improvement in a $25 \mu \mathrm{m}$ diameter glass fiber coated with $100 \mathrm{~nm}$ of platinum relative to a $25 \mu \mathrm{m}$ solid platinum wire is eleven-fold.

Metal-coated $3 \omega$ further reduces previous solid metal $3 \omega$ and THW periodic heating losses at the interface of the heater with bulk electrical connection due to diminished axial thermal conduction proportional to $\sqrt{(k \rho c)_{S} A_{X C}}$, where $(k \rho c)_{s}$ represents the thermal effusivity of the fiber or wire substrate. This approximation of periodic heat through the connector comes from applying 1-D planar periodic heat conduction model, where the 1D axis is through the fiber or solid-wire.

Ideally, all the periodic heat generated from the $3 \omega$ heating element would radially enter the fluid-under-test such that the oscillating temperature depended entirely on the fluid properties. The total heat generated by the heater is split between the fluid and parasitic dissipation mechanisms,

$$
q_{H} e^{i \omega t}=q_{\text {fluid }} e^{i \omega t}+q_{\text {parasitic }} e^{i \omega t} .
$$

While both standard $3 \omega$ and metal-coated $3 \omega$ have parasitic losses of heat into the glass substrate or glass fiber, a greater fraction of the periodic heating enters the fluid with metalcoated $3 \omega$

The ratio of heat entering the glass substrate relative to the fluid for standard $3 \omega$ can at best approach the ratio of the square root of thermal effusivities with an infinitely thin heating element (low frequency limit). ${ }^{25}$

For metal-coated and solid-wire $3 \omega$, the ratio of the periodic heat entering the fluid varies with frequency because the ratio of $L_{p}$ to the fiber diameter changes with frequency. The curves in Fig. 2, based on the conduction model derived in Eqs. (6)-(12), show that more heat enters the fluid for all frequencies in metal-coated $3 \omega$. For metal-coated at very lowfrequency almost all the heat goes into the fluid-under-test, while at very high frequencies, the ratio asymptotes to the standard $3 \omega$ ratio. At very low frequencies, the thermal penetration depth is greater than the fiber diameter, so the heat waves pass through the glass fiber and enter the fluid-undertest relatively undistorted. At high-frequencies, the thermal penetration depth is very short relative to the diameter, and the radial geometry approaches the standard $3 \omega$ ratio. In solid-wire $3 \omega$, heat is periodically generated volumetrically

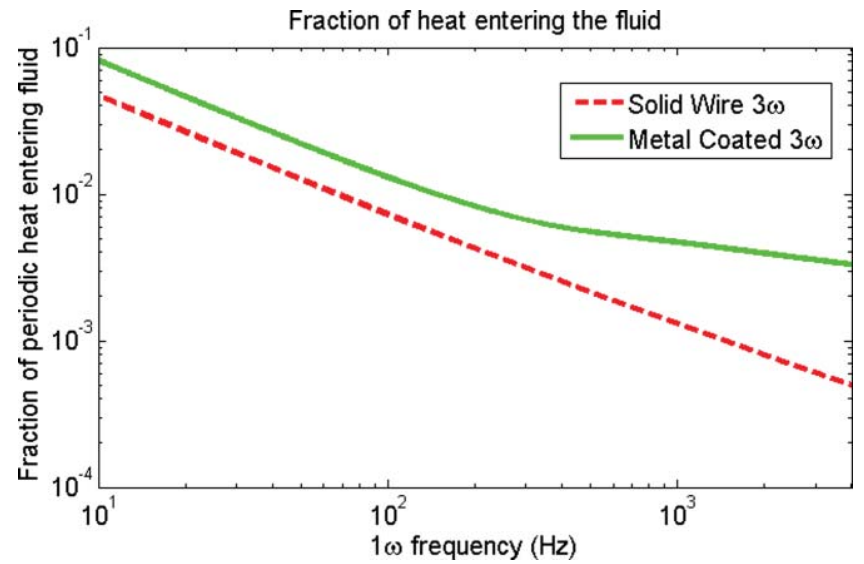

FIG. 2. (Color online) Fraction of zero time-average periodic heating entering fluid-under-test (air). For a metal-coated fiber $(25 \mu \mathrm{m}$ diameter glass fiber coated with $100 \mathrm{~nm}$ of platinum) more of the heat enters the fluid-under-test than with solid metal wire $3 \omega$. Thermoproperties were taken for Pyrex and air at standard temperature and pressure. The analytical model for metal-coated and solid-wire $3 \omega$ are developed in a later section.

throughout the wire, such that thermal oscillations from volumetric heating near the center of the solid-wire may dampen greatly before the thermal wave has traveled to metal-fluid interface. Therefore, the fraction of heat entering the fluid is always smaller than metal-coated and does not asymptote to the standard $3 \omega$ value.

Radiation heat losses can also be ignored without consequence for our validation test on fluids at room temperature, as much less heat is periodically radiated than periodically conducted through the fluid, ${ }^{23}$

$$
\frac{Q_{\text {Rad }}}{Q_{\text {Cond }}}=\frac{8 \varepsilon \sigma T^{3}}{\sqrt{k \rho c \omega}} .
$$

Worst case radiation occurs at high temperature and low frequencies, but will be negligable in our temperature range with the fluids tested.

Another heat transfer mode that must be considered when measuring a fluid is natural convection. The insignificance of natural convection to metal-coated $3 \omega$ is shown by the steadystate natural convection fluid speed being order of magnitudes slower than the conduction wave propagation speed. Natural convection speeds were estimated as $u_{N C} \sim(g \beta \Delta T L)^{1 / 2}$, and the conduction wave velocity was estimated as product of thermal wavelength and frequency, $v_{\text {Condwave }}=L_{P} \omega$ $=\sqrt{2 \alpha \omega} \cdot{ }^{25}$ For air, $u_{N C}$ is similar in magnitude to $v_{\text {Cond Wave }}$ only when the heating frequency is far less than $1 \mathrm{~Hz}$. Our measurements are typically taken at $10-1000 \mathrm{~Hz}$.

\section{Manufacturing technique}

The manufacturing of the metal-coated glass fiber has two distinct steps, coating a glass fiber in metal and electronically mating the metal-coated fiber to a bulk electrical connector.

To adapt a conventional sputtering machine (Perkin Elmer $6 \mathrm{~J}$ ) to coat a cylinder uniformly, fibers were strung onto a spool, which was then rotated with a vacuum motor (Fig. 3(a)). This "sputtering lathe" evenly coated the fiber in 
(1)

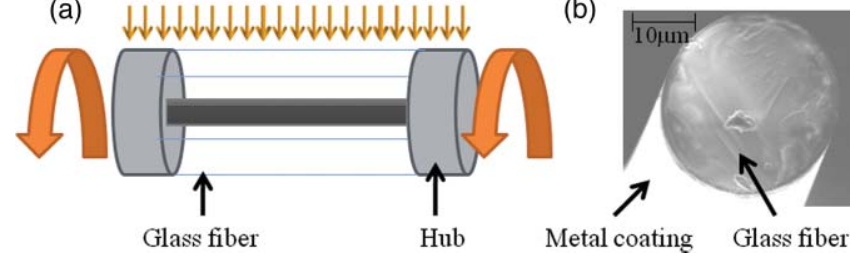

FIG. 3. (Color online) (a) "Sputtering lathe" - glass fibers are strung across spool which rotated during metal deposition to ensure circumferentially even coating. Glass fibers are adhered to the spool with Kapton tape. (b) Metalcoated fiber cross-section scanning electron microscope image.

metal, as the rotation period was much shorter than the sputtering time. A $10 \mathrm{~nm}$ titanium adhesion layer and 50-100 nm of gold or platinum were applied to the fiber. To convert from standard planar sputtering deposition rates to "sputtering lathe" deposition rates, the rates were cut by a factor of $\pi$. This ratio stems from the ratio of the fiber's areal footprint (i.e., its diameter times its length), to its actual surface area (i.e., its circumference times its length).

The raw glass fiber, used as the backbone of the metalcoated fiber, has a 1/1000 in. diameter and was supplied by Fiberoptics Technologies, Inc. (0.125 in. $\times 30$ in. Raw Fiber Bundle, 0.66 NA, 0.001 in. Fiber Diameter, FTIRF12753). This fiber consists of a leaded glass core (Schott F2) and borosilicate cladding (Schott 8250), shown in Fig. 3(b). The diameters and properties of the two different glass sections were provided by the supplier and are included in Table II. Bulk electrical contact was made with the metal-coated fiber using EpoTech H27D, silver electrically conducting epoxy $(\rho \leq 0.0005 \Omega / \mathrm{cm} @ 300 \mathrm{~K})$ without tensioning the fiber. The epoxy was cured at $80^{\circ} \mathrm{C}$ for $24 \mathrm{~h}$. Measurements were insensitive to the length of fiber; measurements for this benchmark study were performed with a fiber roughly $5 \mathrm{~mm}$ in length, but both shorter $(2 \mathrm{~mm})$ and longer $(20 \mathrm{~mm})$ lengths were used with air without significant differences. Insensitivity to the fiber length, and hence resistance, indicates that the resistance of the epoxy and leads does not detrimentally impact our results.

\section{ELECTRONIC CONFIGURATION}

The experiment is performed by immersing the fiber in the fluid under test and driving a periodic current across it while measuring the resultant voltage. The current sourced by a Keithley $6221 \mathrm{DC}$ and AC Current Source passes in series through the metal-coated fiber and a calibration resistor (Fig. 4). The calibration resistor is tuned to equal the nominal resistance of the metal-coated resistor during operation. The voltage across the sense and calibration resistors are buffered by an instrumentation amplifier (LT 1167). The instrumentation amplifier outputs into the lock-in amplifier (Stanford Research Systems SR830). The lock-in amplifier performs a real-time Fourier decomposition of the signal. To remove noise introduced by the current source, the lock-in amplifier analyzes the difference in voltage between the metal-coated fiber and calibration resistors (A-B Mode). The amplitude and phase of the third harmonic $(3 \omega)$ signal is collected via a MATLAB program that interfaces with the

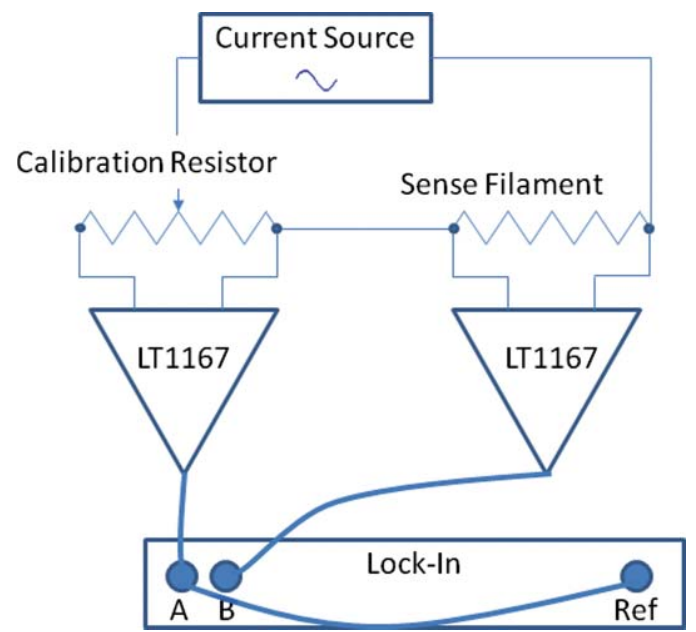

FIG. 4. (Color online) $3 \omega$ electronic schematic: Current source provides a $1 \omega$ current to the series circuit consisting of the metal-coated filament and the calibration resistor. The calibration resistor is adjusted such that it equals the average sense filament resistance. The voltages across the metal-coated filament and calibration resistor are buffered by an instrumentation amplifier and fed into a lock-in amplifier. The lock-in determines the magnitude and phase of the third-harmonic $(3 \omega)$ voltage, thereby measuring the magnitude and phase of the metal coating's temperature.

electronics. The MATLAB program sweeps through a range of frequencies and records the magnitude and phase of the $3 \omega$ signal, which is the input used to determine the thermal conductivity of the fluid under test.

\section{A. Generalized solution for determination of thermal conductivity}

The thermal conductivity of the unknown fluid is determined by fitting phase and amplitude data with the analytical solution to the heat conduction equation, using $k_{\text {fluid }}$ as the sole fitting parameter. The fit is optimized using least-squares minimization. We now present a generalized solution for an $n$-layer concentric cylindrical structure with heat generated periodically in one or more layers. is $^{27}$

The heat conduction equation in cylindrical coordinates

$$
k_{n} \frac{1}{r} \frac{\partial}{\partial r}\left(r \frac{\partial T_{n}}{\partial r}\right)+q_{n}^{\prime \prime \prime}=\rho_{n} c_{n} \frac{\partial T_{n}}{\partial t},
$$

where $n$ indexes the layer. The multilayer metal-coated $3 \omega$ model has $q_{n}^{\prime \prime \prime}=0$, except in the metal layer, where

$$
q_{n}^{\prime \prime \prime}=q_{0}^{\prime \prime \prime} e^{i \omega t}
$$

This set of equations has solutions in the form below, where $T_{n}$ indicates magnitude and phase of temperature oscillations,

$$
\begin{gathered}
T_{n}(r, t)=e^{i \omega t}\left(C_{n, I} I_{0}\left(\gamma_{n} r\right)+C_{n, K} K_{0}\left(\gamma_{n} r\right)+\frac{\alpha_{n} q_{n}^{\prime \prime \prime}}{i \omega k_{k}}\right), \\
\gamma_{n}^{2}=\frac{i \omega}{\alpha_{n}},
\end{gathered}
$$

and boundary conditions at each interface are

$$
\begin{gathered}
-\left.k_{n} \frac{\partial T_{n}}{\partial r}\right|_{r=r_{n}}=-\left.k_{n+1} \frac{\partial T_{n+1}}{\partial r}\right|_{r=r_{n}}, \\
-\left.k_{n} \frac{\partial T_{n}}{\partial r}\right|_{r=r_{n}}=h_{n}\left(T_{n}\left(r_{n}\right)-T_{n+1}\left(r_{n}\right)\right),
\end{gathered}
$$


where $h_{n}$ is the thermal boundary conductance.

Symmetry at $r=0$, leads to no heat conduction through the center,

$$
\left.\frac{\partial T_{1}}{\partial r}\right|_{r \rightarrow 0}=0
$$

This condition arises because as $r \rightarrow 0$ volume goes to 0 , so if any heat was entering or exiting, there would be a non-physical infinite temperature reached.

$$
\text { At } r \rightarrow \infty, T_{m}(r \rightarrow \infty)=0 .
$$

This condition forces the outermost layer's temperature oscillation magnitude to approach zero as radius goes to infinity.

The coefficients preceding the modified Bessel functions in each layer can be solved by formulating the boundary conditions in terms of a set of linear equations. The first layer has

only a coefficient $C_{1 I}$, as $C_{1 K}$ is zero due to the $r \rightarrow 0$ boundary condition. Likewise, the last layer has only the coefficient $C_{\text {LastK }}$ because $C_{\text {LastI }}$ is zero from the $r \rightarrow \infty$ boundary condition. All other layers have two coefficients and therefore a matrix $[A]$ will be defined. $[A]$ is a square matrix with size $2 l$ $\times 2 l$, where $l$ is the number of interfaces. Likewise, the $\bar{x}$ will have one column and $2 l$ rows,

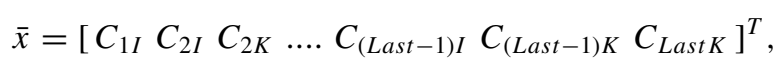

$$
[A]=\left[\begin{array}{cccc}
A_{1,1} & A_{1,2} & \ldots & A_{1, l * 2} \\
A_{2,1} & A_{2,2} & \ldots & \\
\vdots & & \ddots & \\
A_{l * 2,1} & & & A_{l * 2, l * 2}
\end{array}\right]
$$

For interface, $l=1$ :

$A_{1,1}=-k_{1} \gamma_{1} I_{1}\left(\gamma_{1} r_{1}\right)$,

$A_{1,2}=k_{2} \gamma_{2} I_{1}\left(\gamma_{2} r_{1}\right)$,

$A_{1,3}=-k_{2} \gamma_{2} K_{1}\left(\gamma_{2} r_{1}\right)$,

$A_{2,1}=h_{1} I_{0}\left(\gamma_{1} r_{1}\right)+k_{1} \gamma_{1} I_{1}\left(\gamma_{1} r_{1}\right)$,

$A_{2,2}=-h_{1} I_{0}\left(\gamma_{2} r_{1}\right)$,

$A_{2,3}=-h_{1} K_{0}\left(\gamma_{2} r_{1}\right)$,

$b_{2,1}=i h_{1}\left(\alpha_{2} q_{2} / k_{2}-\alpha_{1} q_{1} / k_{1}\right) / \omega$
For interfaces, $l \geq 2, l \neq$ last :

$A_{l * 2-1, l * 2-2}=-k_{l} \gamma_{l} I_{1}\left(\gamma_{l} r_{l}\right)$,

$A_{l * 2-1, l * 2-1}=k_{l} \gamma_{l} K_{1}\left(\gamma_{l} r_{l}\right)$,

$A_{l * 2-1, l * 2}=k_{l+1} \gamma_{l+1} I_{1}\left(\gamma_{l+1} r_{l}\right)$

$A_{l * 2-1, l * 2+1}=-k_{l+1} \gamma_{l+1} K_{1}\left(\gamma_{l+1} r_{l}\right)$

For interface, $l=$ last :

$A_{l * 2-1, l}=-k_{l} \gamma_{l} I_{1}\left(\gamma_{l} r_{l}\right)$,

$A_{l * 2-1, l+1}=k_{l} \gamma_{l} K_{1}\left(\gamma_{l} r_{l}\right)$,

$A_{l * 2-1, l+2}=-k_{l+1} \gamma_{l+1} K_{1}\left(\gamma_{l+1} r_{l}\right)$,

$A_{l * 2, l * 2-2}=h_{l} I_{0}\left(\gamma_{l} r_{l}\right)+k_{l} \gamma_{l} I_{1}\left(\gamma_{l} r_{l}\right), \quad A_{l * 2, l}=h_{l} I_{0}\left(\gamma_{l} r_{l}\right)+k_{l} \gamma_{l} I_{1}\left(\gamma_{l} r_{l}\right)$,

$A_{l * 2, l * 2-1}=h_{l} K_{0}\left(\gamma_{l} r_{l}\right)-k_{l} \gamma_{l} K_{1}\left(\gamma_{l} r_{l}\right), \quad A_{l * 2, l+1}=h_{l} K_{0}\left(\gamma_{l} r_{l}\right)-k_{l} \gamma_{l} K_{1}\left(\gamma_{l} r_{l}\right)$,

$A_{l * 2, l * 2}=-h_{l} I_{0}\left(\gamma_{l+1} r_{l}\right)$,

$A_{l * 2, l * 2+1}=-h_{l} K_{0}\left(\gamma_{l+1} r_{l}\right)$,

$A_{l * 2, l+2}=-h_{l} K_{0}\left(\gamma_{l+1} r_{l}\right)$,

$b_{l * 2,1}=i h_{l}\left(-\alpha_{l} q_{l} / k_{l}\right) / \omega$

$b_{l * 2,1}=i h_{l}\left(\alpha_{l+1} q_{l+1} / k_{l+1}-\alpha_{l} q_{l} / k_{l}\right) / \omega$

$$
[A] \bar{x}=b \Rightarrow \bar{x}=[A]^{-1} b .
$$

By inverting $[A]$ and solving for the $\mathrm{x}$-vector, the constants for each layer can be solved. These constants can then be applied to the previous general solution for temperature in a layer. The amplitude and phase averaged over the thickness of the metal heating layer were fit to data. Fits were optimized by varying the thermal conductivity of the unknown fluid - the value resulting in the least-squares fit with data is the reported value of thermal conductivity. In a similar manner thermal boundary conductance or specific heat, rather than thermal conductivity, can be solved with least-squares minimization. Like all $3 \omega$ methods, fitting to more than one variable at the same time increases the uncertainty in both predictions.

\section{RESULTS AND DISCUSSION}

To validate the metal-coated $3 \omega$ technique benchmark fluids having thermal conductivities spanning nearly 2 orders of magnitude were tested. Current frequencies from $10-500 \mathrm{~Hz}$ were used creating $L_{P}$ on the order of $1.5 \mathrm{~mm}$ to $0.2 \mathrm{~mm}$ for air at standard temperature and pressure. While higher frequencies could be accessed, this range still enabled measurement in minutes, rather than over a $24 \mathrm{~h}$ period as required by the solid-wire experiment. ${ }^{9}$ The benchmarks include argon, air, helium, and deionized water. Figure 5 compares the normalized $\Delta T$ and phase lag between fluids of various thermal conductivities. Normalization of temperature oscillation magnitude was performed by dividing the raw $\Delta V_{3 \omega}$ signal at all frequencies by the maximum $\Delta V_{3 \omega}$ occurring at the lowest measured frequency. The shape of the normalized amplitude plot depends on thermal conductivity and monotonically decreases for all materials. The phase signal, which is unaffected by normalization, changes more dramatically between the different gases. Note that while in magnitude argon and helium lie very close to each other, in phase they are distinguishable. By using normalized amplitude and phase we avoid the need for direct knowledge of $\beta$, which affects only the absolute magnitude of $\Delta T$. MATLAB's nlinfit function was used to determine the thermal conductivity of the fluid that minimized the square error between the experimental data and analytical solution.

The generalized solution presented earlier was used to fit experimental data (normalized amplitude and phase) which yielded thermal conductivities in agreement with Refs. 20 and 25 . These values are listed in Table I with associated uncertainties. The dimensions and properties of our metal coated 

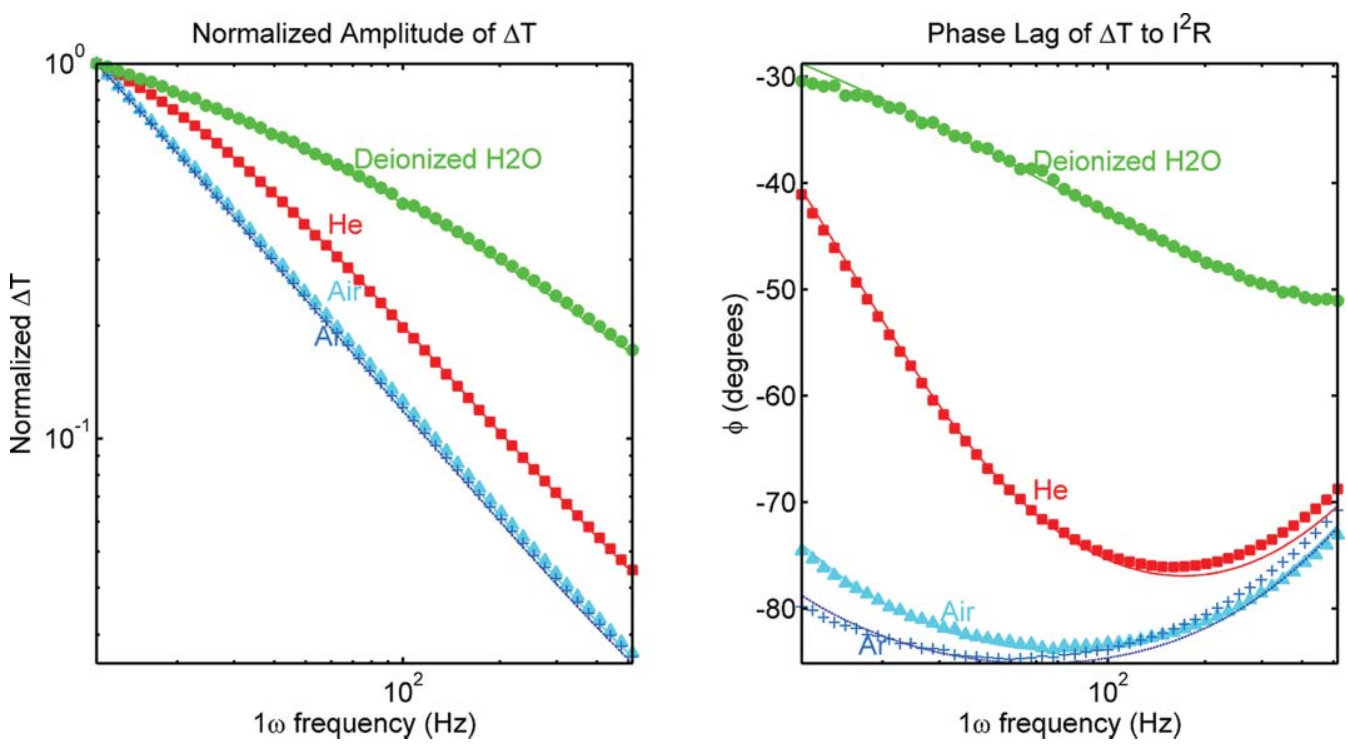

FIG. 5. (Color online) Normalized amplitude and phase versus frequency for various fluids (Argon - dark blue cross, Air - light blue triangle, Helium - red square, Deionized $\mathrm{H}_{2} \mathrm{O}$ - green circles) with corresponding analytical fits shown as similarly colored lines. The experimental and accepted thermal conductivities are shown in Table I. The data was normalized by dividing a given fluid's $\Delta$ Ts by its maximum $\Delta \mathrm{T}$. This normalization is optional, but removes the need for measuring the heater's coefficient of thermoresistance. A least-squares minimization was then used to fit experimental data to analytical model.

fiber, which were assumed in the analytical model, are shown in Table II. This model includes four layers $(n=4)$ to account for the leaded glass core, the borosilicate cladding, the metal coating, and the surrounding sample. Uncertainties were calculated by propagating the uncertainties in parameters input to the analytical solution. Their impacts on the uncertainty were combined orthogonally $\left(\Delta k=\sqrt{\sum_{i} \Delta k_{i}^{2}}\right.$, where $\Delta k_{i}$ is the uncertainty in $k$ due to parameter $i$ ). We find that this solution is most sensitive to fiber diameter.

Using the analytical models validated by our benchmark of metal-coated $3 \omega$, anticipated $\Delta T$ amplitude and phase were calculated for a range of thermal boundary conductances. In the metal-coated $3 \omega$, the phase shape versus frequency is significantly affected by thermal boundary conductance (Fig. 6). In this figure, the change in phase for a decade of thermal boundary conductance (between $10^{7}$ and $10^{8} \mathrm{~W} / \mathrm{m}^{2} \mathrm{~K}$ ) is plotted for metal-coated and solid-wire $3 \omega$, surrounded by liquid $\mathrm{Hg}$. This change in phase is greater for metal-coated $3 \omega$. Changing the probe-Hg thermal boundary resistance from $10 \mathrm{MW} / \mathrm{m}^{2} \mathrm{~K}$ to $100 \mathrm{MW} / \mathrm{m}^{2} \mathrm{~K}$ results in an average phase difference of about $2^{\circ}$ with metal-coated $3 \omega$, and only $0.7^{\circ}$ with solid-wire $3 \omega$. The sensitivity to thermal boundary conductance shown here is enhanced by using

TABLE I. Comparison of accepted thermal conductivity values (Refs. 20 and 25) to experimental values obtained with this experiment on benchmark fluids. Experiments performed at atmospheric pressure at $300 \mathrm{~K}$.

\begin{tabular}{lcccc}
\hline \hline & $\begin{array}{c}k_{\text {Experimental }} \\
(\mathrm{W} / \mathrm{m} \mathrm{K})\end{array}$ & $\pm k \%$ & $\begin{array}{c}\Delta k \\
(\mathrm{~W} / \mathrm{m} \mathrm{K})\end{array}$ & $\begin{array}{c}k_{\text {Accepted }} \\
(\mathrm{W} / \mathrm{m} \mathrm{K})\end{array}$ \\
\hline Deionized $\mathrm{H}_{2} \mathrm{O}$ & 0.610 & $4.19 \%$ & 0.026 & 0.593 \\
$\mathrm{He}$ & 0.163 & $5.04 \%$ & 0.008 & 0.156 \\
Air & 0.027 & $5.17 \%$ & 0.001 & 0.026 \\
Argon & 0.018 & $8.61 \%$ & 0.002 & 0.017 \\
\hline \hline
\end{tabular}

a high thermal conductivity fluid and sensing at higher frequencies. The validation samples and frequency ranges were insensitive to thermal boundary conductance.

\section{CONCLUSIONS}

A modified $3 \omega$ technique that employs a suspended glass fiber coated with metal, used as a heater/thermometer, has been developed. This technique was successfully benchmarked against liquid and gas samples spanning 2-orders of magnitude in thermal conductivity. The benefits of this technique are larger signal strength for a given $\Delta T$, less parasitic conduction loss, no need for absolute calibration of $\Delta T$, rapid measurement, and enhanced sensitivity to thermal boundary

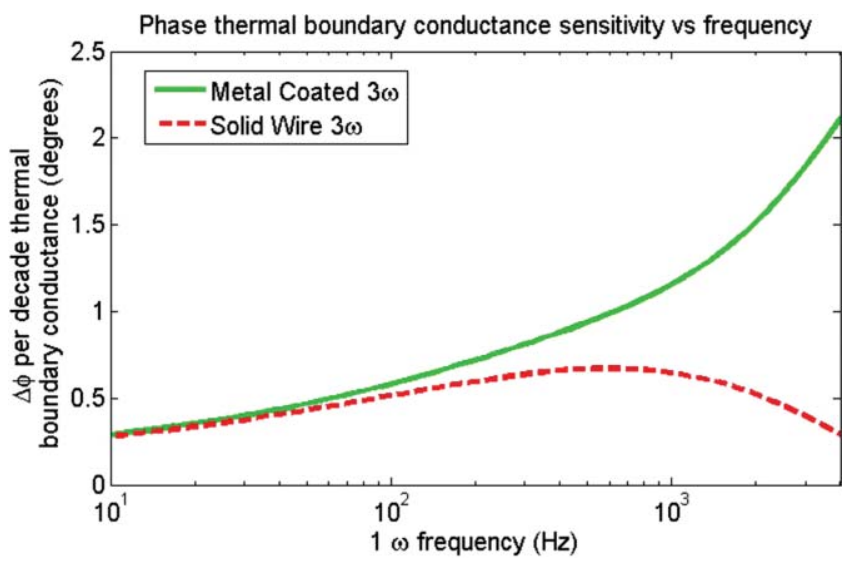

FIG. 6. (Color online) A simulated plot of change in phase between thermal boundary conductance of $10^{7}$ and $10^{8} \mathrm{~W} / \mathrm{m}^{2} \mathrm{~K}$ in metal-coated $3 \omega$ (solid green) and solid metal $3 \omega$ (dashed red). The greater separation between metal-coated $3 \omega$ curves indicates greater sensitivity to thermal boundary conductance than solid metal $3 \omega$. Simulated experiment has heater surrounded by mercury with a thermal boundary conductance across metal-mercury interface. 
TABLE II. Properties and dimensions of experimental setup assumed for the predictions of thermal conductivity shown in Table I. Accepted values of $\rho$ and $c$ were used for each fluid sample (Refs. 20 and 25).

\begin{tabular}{lcccc}
\hline \hline & $\begin{array}{c}\text { Layer } n=1 \\
\text { (Schott } F 2^{\mathrm{a}} \text { leaded glass) }\end{array}$ & $\begin{array}{c}\text { Layer } n=2 \\
\text { (Schott } 8250^{\mathrm{a}} \text { borosilicate) }\end{array}$ & $\begin{array}{c}\text { Layer } n=3 \\
\text { (Platinum) }\end{array}$ & $\begin{array}{c}\text { Layer } n=4 \\
\text { (Sample) }\end{array}$ \\
\hline$r_{n}(\mu \mathrm{m})$ & 12 & 13.1 & 13.15 & $\ldots$ \\
$k_{n}(\mathrm{~W} / \mathrm{m} \mathrm{K})$ & 0.78 & 1.18 & 71.6 & Table I \\
$\rho_{n}\left(\mathrm{~kg} / \mathrm{m}^{3}\right)$ & 3600 & 2280 & 21450 & See Refs. 20 and 25 \\
$c_{n}(\mathrm{~J} / \mathrm{kg} \mathrm{K})$ & 557 & 720 & 130 & See Refs. 20 and 25 \\
\hline \hline
\end{tabular}

${ }^{\text {a }}$ Properties and dimensions were supplied by the manufacturer.

conductance. Relative to existing $3 \omega$ approaches for measuring fluids, this means measurement times are reduced from hours to minutes, sample sizes are reduced from tens-ofcentimeters to millimeters, and low thermal effusivity materials are measurable where they have not been before.

\section{ACKNOWLEDGMENTS}

This work was supported by the AFOSR Young Investigator Research Program. We thank the National Science Foundation (NSF) REU Summer Program and undergraduate summer research assistant David Ring. S.N.S. thanks the Carnegie Mellon Steinbrenner Fellowship.

${ }^{1}$ L. Lu, W. Yi and D. L. Zhang, Rev Sci Instrum 72(7), 2996 (2001).

${ }^{2}$ L. Shi, D. Y. Li, C. H. Yu, W. Y. Jang, D. Kim, Z. Yao, P. Kim, and A. Majumdar, ASME Trans. J. Heat Transfer 125(5), 881 (2003).

${ }^{3}$ F. Chen, J. Shulman, Y. Xue, C. W. Chu, and G. S. Nolas, Rev. Sci. Instrum. 75(11), 4578 (2004).

${ }^{4}$ S. R. Choi, J. Kim, and D. Kim, Rev. Sci. Instrum. 78(8), 084902 (2007).

${ }^{5}$ Z. L. Wang, D. W. Tang, and W. G. Zhang, J. Phys. D: Appl. Phys. 40(15), 4686 (2007)

${ }^{6}$ H. C. Chien, D. J. Yao, M. J. Huang, and T. Y. Chang, Rev. Sci. Instrum. 79(5), 054902 (2008).

${ }^{7}$ S. R. Choi and D. Kim, Rev. Sci. Instrum. 79(6), 064901 (2008).

${ }^{8}$ J. Buongiorno, D. C. Venerus, N. Prabhat, T. McKrell, J. Townsend, R. Christianson, Y. V. Tolmachev, P. Keblinski, L. W. Hu, J. L. Alvarado, I. C. Bang, S. W. Bishnoi, M. Bonetti, F. Botz, A. Cecere, Y. Chang, G. Chen, H. S. Chen, S. J. Chung, M. K. Chyu, S. K. Das, R. Di Paola, Y. L. Ding, F. Dubois, G. Dzido, J. Eapen, W. Escher, D. Funfschilling, Q. Galand, J. W. Gao, P. E. Gharagozloo, K. E. Goodson, J. G. Gutierrez, H. P. Hong, M. Horton, K. S. Hwang, C. S. Iorio, S. P. Jang, A. B. Jarzebski, Y. R. Jiang,
L. W. Jin, S. Kabelac, A. Kamath, M. A. Kedzierski, L. G. Kieng, C. Kim, J. H. Kim, S. Kim, S. H. Lee, K. C. Leong, I. Manna, B. Michel, R. Ni, H. E. Patel, J. Philip, D. Poulikakos, C. Reynaud, R. Savino, P. K. Singh, P. X. Song, T. Sundararajan, E. Timofeeva, T. Tritcak, A. N. Turanov, S. Van Vaerenbergh, D. S. Wen, S. Witharana, C. Yang, W. H. Yeh, X. Z. Zhao, and S. Q. Zhou, J. Appl. Phys. 106(9), 094312 (2009).

${ }^{9}$ S. M. Lee, Rev. Sci. Instrum. 80(2), 024901 (2009).

${ }^{10}$ E. Yusibani, P. L. Woodfield, M. Fujii, K. Shinzato, X. Zhang, and Y. Takata, Int. J. Thermophys. 30(2), 397 (2009).

${ }^{11}$ E. Pop, S. Sinha, and K. E. Goodson, Proc. IEEE 94(8), 1587 (2006)

${ }^{12}$ E. S. Landry and A. J. H. McGaughey, Phys. Rev. B 79(7), 075316 (2009).

${ }^{13}$ R. Venkatasubramanian, Phys. Rev. B 61(4), 3091 (2000).

${ }^{14}$ M. S. Dresselhaus, G. Chen, M. Y. Tang, R. G. Yang, H. Lee, D. Z. Wang, Z. F. Ren, J. P. Fleurial, and P. Gogna, Adv. Mater. 19(8), 1043 (2007).

${ }^{15}$ Y. Rabin, CryoLetters 21(3), 163 (2000).

${ }^{16}$ A. Schmidt, M. Chiesa, X. Y. Chen, and G. Chen, Rev. Sci. Instrum. 79(6), 064902 (2008).

${ }^{17}$ B. Klaudinyi, E. A. Snajdr, and J. R. Friedrichs, Am. Ceram. Soc. Bull. 55(8), 739 (1976).

${ }^{18}$ E. V. Somers and J. A. Cyphers, Rev. Sci. Instrum. 22(8), 583 (1951).

${ }^{19}$ K. D. Maglic, A. Cezairliyan, and V. E. Peletsky, Compendium of Thermophysical Property Measurement Methods (Plenum, New York, 1984).

${ }^{20}$ D. R. A. Perkins, NIST Experimental Property of Fluids Group, Vol. 2010.

${ }^{21}$ J. J. Healy, J. J. Degroot, and J. Kestin, Physica B \& C 82(2), 392 (1976).

${ }^{22}$ Y. Nagasaka and A. Nagashima, Rev. Sci. Instrum. 52(2), 229 (1981).

${ }^{23}$ D. G. Cahill, Rev. Sci. Instrum. 61(2), 802 (1990).

${ }^{24}$ C. Dames and G. Chen, Rev Sci Instrum 76(12), 124902 (2005).

${ }^{25}$ A. F. Mills, Heat transfer, 2nd ed. (Prentice Hall, Upper Saddle River, NJ, 1999).

${ }^{26}$ R. Heyd, A. Hadaoui, M. Fliyou, A. Koumina, L. E. Ameziane, A. Outzourhit, and M. L. Saboungi, Rev. Sci. Instrum. 81(4), 044901 (2010).

${ }^{27}$ H. S. Carslaw and J. C. Jaeger, Conduction of heat in solids, 2nd ed. (Clarendon, Oxford, 1959). 